\title{
THE PREDICTIVE VALIDITY OF SEVEN MANUAL DEXTERITY TESTS
}

\author{
Y. RIM \\ TECHNION - Israel Instinte of Technology
}

This study was planned to find out whether manual dexterity tests can predict success of high school students in shop mechanics in a Vocational High School.

The training in operations requiring manual dexterity is an important part of the curriculum of the Vocational High School whose first-year students served as subjects in the present investigation. The program of machine shop training for students of the first year includes the following operations: filing; sawing; chipping; measuring; layout; drilling; scraping; grinding (drills, chisels, etc.); fitting; riveting; turning; assembling. It is, therefore, very important to select among candidates those who will succeed in learning to perform these operations.

What type of ability is needed to perform successfully the above mentioned operations? Too little is known about the structure of manual ability to enable us an a priori selection of tests. Is two-hand coordination more important than eye-hand coordination? What is the place of the speed and accuracy of arm and hand movement in the hierarchy of manual dexterities?

It was felt that the performance of students at the beginning of their first year at a Vocational High School would reflect aptitude rather than achievement. In this period the first six of the above mentioned operations were taught and trained. It was therefore decided to administer seven well-known tests of manual dexterity to first-year students in an attempt to find out which tests would best predict success in shop mechanics.

\section{PROCEDURE}

Population. Ss were 224 first-year students at the Vocational High School attached to the Technion-Israel Institute of Technology in Haifa.

Tests used. The following tests were administered:

A. Pennsylvania Bi-manual Worksample (8)

B. Purdue Pegboard (5)

C. Crawford Small Parts Dexterity (4)

D. Minnesota Rate of Manipulation (6)

E. Bennett Hand-Tool Dexterity (2)

F. Stromberg Dexterity (10)

G. O'Connor Tweezer and Finger Dexterity (7)

The tests were administered according to instructions given in the respective Examiner's Manuals. Of the Minnesota Rate of Manipulation Test the first three subtests only were administered: Placing, Turning and Displacing.

Scores on two paper-and-pencil tests were also available;

1) A non-verbal test of intelligence of the Dominoes-type.

* Thanks are due to Mr. S. Zacks of the Department of Industrial Engineering and Management for his help extended in the statistical treatment of the data. 
2) A Mechanical Comprehension Test of the Bennett-type.

Design. The tests were administered in the order shown below to seven groups of 32 students each, so that each group was given two tests, and each test was given to two groups.

The order in which the tests were administered:

$\begin{array}{llllllll}\text { Group } & \text { I } & \text { II } & \text { III } & \text { IV } & \text { V } & \text { VI } & \text { VII } \\ \text { Tests } & \text { AE } & \text { EF } & \text { FG } & \text { GB } & \text { BC } & \text { CD } & \text { DA }\end{array}$

For each group of 64 students three tests were accordingly available: Dominoes, Mechanical Comprehension, and one of the seven dexterity tests.

Criteria: a) Grades received after three months' training served as criterion.

b) Grades received after the end of schoolyear.

\section{Results}

Table 1 summarizes the results after three months' instruction; Table 2 summarizes the results at the end of the schoolyear. The consistency of the results over the whole year are striking.

Multiple correlations were calculated between scores received on Dominoes and Mechanical Comprehension and the shop grades received after 3-months' instruction and experience in performance of the six operations: filing, sawing, chipping, measuring, layout and drilling. (See col. Ro of Table I).

Table 1. Multiple Correlations between Paper-and-Pencil Tests, Seven Manual Dexterity Tests, and Grades in Shop Mechanics. (after three months' instruction).

\begin{tabular}{c|ccc}
\hline Test & Ro & R & $\frac{\text { R-Ro }}{\text { Ro }} \times 100$ \\
\hline A & .373 & .377 & 1.0 \\
B & .330 & .725 & 119.0 \\
C & .228 & .355 & 55.7 \\
D & .190 & .810 & 326.0 \\
E & .319 & .414 & 29.7 \\
F & .267 & .387 & 44.9 \\
G & .517 & .577 & 11.6 \\
\hline
\end{tabular}

Table 2. Multiple Correlations between Paper-and-Pencil Tests, Seven Manual Dexterity Tests, and Grades in Shop Mechanics. (at the end of schoolyear)

\begin{tabular}{c|ccc}
\hline Test & Ro & R & $\frac{\text { R-Ro }}{\text { Ro }} \times 100$ \\
\hline A & .384 & .395 & 3.0 \\
B & .340 & .744 & 118.8 \\
C & .240 & .366 & 52.5 \\
D & .200 & .820 & 310.0 \\
E & .291 & .422 & 45.0 \\
F & .256 & .377 & 47.2 \\
G & .487 & .566 & 16.2 \\
\hline
\end{tabular}

For the groups which took tests A, B, C, D, E, F, the multiple correlations values were of the same order of magnitude. However, the multiple correlation for the group which took test $\mathrm{G}$ differed significantly from the other values. No explanation is offered, since the students constituting the seven groups were chosen at random from the various classrooms. 
Multiple R's were then calculated between the shop grades and the scores on Dominoes, Mechanical Comprehension and each test of dexterity (See col. $R$ of Table 1). These multiple R's are always greater than those of column Ro. The excess of the multiple R's for all the tests over those for Dominoes and Mechanical Comprehension alone, measured the contribution of the dexterity test to the predictability value. Column three gives an estimate of the contribution of each test to the prediction of success in Shop Mechanics.

\section{Discussion}

It can be seen from Table 1 that the order in which the seven tests of dexterity contributed to the prediction of success in Shop Mechanics over and above the paper-andpencil tests is as follows:

1. Minnesota Rate of Manipulation

2. Purdue Pegboard

3. Crawford Small Parts Dexterity

4. Stromberg Dexterity

5. Bennett Hand-Tool Dexterity

6. O'Connor Tweezer and Finger Dexterity

7. Pennsylvania Bi-manual Worksample

In what way do the results contribute to our insight of the structure of abilities needed to succeed in performing the necessary operations leading to superior grades in Shop Mechanics?

The factor analysis carried out by the United States Employment Service (9) revealed two dexterity factors, one of which was common to the Placing and Turning tests of the Minnesota Rate of Manipulation Test and the Pegboard Apparatus of the USES (both of which require relatively gross movements). The Placing and Turning tests have practically identical factor composition; they are almost pure tests of arm-and-hand dexterity.

In his discussion of the nature and role of manual dexterities, Super (11) drew our attention to the unique contribution made by Cohen and Strauss (3) to the understanding of manual skill and the nature of semiskilled work.

"It was found that in general, the more skilled operatives (so classified by a standard time-and-motion study technique) performed their work more simply.... The more proficient operatives make the movements of their two hands overlap more than the less proficient workers, thus performing two operations at once instead of one after the other. The Purdue Pegboard . . . is almost unique in testing this type of two-hand coordination" (11).

Work done at the Medical Research Council's Applied Research Unit in Cambridge, England, made it even clearer "that by far the most important characteristic of expert bodily skill is "timing" . . Efficiency depends . . upon the regulation of the flow from component to component in such a way that now here in the whole series is there any appearance of hurry, and nowhere unnecessarily prolonged derdy" (1).

The Crawford Small Parts Dexterity Test, which claims to measure eyc-hand coordination is the third test contributing substantially to the predict of success.

Thus, the three tests contributing most to the prediction of success, suggest that the 
components of the ability needed constitute a hierarchical order:

a) arm-and-hand dexterity (gross movements) as measured by the Minnesota Rate of Manipulation Test.

b) two-hand co-ordination, as measured by the Purdue Pegboard.

c) eye-hand coordination, as measured by the Crawford Small Parts Dexterity Test.

\section{Summary and Conclusions}

The present study was planned to find out whether manual dexterity tests can predict success of students in Shop Mechanics in a Vocational High School. Seven well-known tests were administered to first-year students. (i.c.- their ninth school year) at the beginning of the year. Some tests were found to contribute significantly to the prediction of success. The results suggest that the most important components of the ability needed arc

1. arm-and-hand dexterity as tested by the Minnesota Rate of Manipulation Test and to a lesser extent by Stromberg Dexterity Test.

2. two-hand coordination as tested by the Purdue Pegboard.

3. eye-hand coordination as tested by the Crawford Small Parts Dexterity.

The results suggests that grades received at the end of three month' instruction and training in Shop Mechanics already reflect the differences between more and less proficient students, as measured by the best discriminating dexterity tests.

Some doubts are raised from time to time with regards to the cross-cultural applicability of tests. This study, carried out in Israel, seems to allay these doubts at least for the use of dexterity tests.

\section{REFERENCES}

1. Bartlett, Sir F. Thinking London: George Allen, 1958. p. 15.

2. Bennett, C. K. Manual of directions for the HandTool Test. New York: Psychological Corp.

3. Cohen, L. \& Strauss, L. Time study and the fundamental nature of manual skill. $J$. consult. Psychol., 1946, 10, 146-153.

4. Crawford, J. E. \& D. M. Crawford small parts dexterity test. Revised Manual. New York: Psychological Corp., 1956.

5. Examiner Manual for the Purdue Pegboard. Chicago: Science Research Associates, 1948.

6. Minnesota Rate of Manipulation Test. Examiner's Manual. Minneapolis: Educ. Test Bureau, Edu- cational Publishers, Inc., 1957.

7. O'Connor Finger and Tweezer Dexterity Tests.Examiner's Manual. Chicago: C. H. Stoelting Co.

8. Roberts, J.R. Pennsylvania bi-manual worksample. Examiner's Manual. Minneapolis: Educ. Test Bureau, Educational Publishers, 1945.

9. Staff, Division of Occupational Analysis W. M. C. Factor analysis of occupational aptitude tests. Educ. Psychol. Measmt., 1945, 5, 147-155.

10. Stromberg, E. L. Stromberg dexterity test. Preliminary Manual. New York: Psychological Corp., 1951.

11. Super, D.E. Appraising occupational fitness. New York: Harper, 1949. P. 186.

MS. received VI 20,61 .

Y. Rim (1922-) Student of the late Prof. Bonaventura at the Hebrew Univ., Jerusalem; 1953 Ph. D. (Univ. of London, Institute of Psychiatry); 1956- lecturer, Technion-Israel Institute of Technology, Technion City, Israel. 